\title{
Population Data on Finland 1900-2004 ${ }^{1}$
}

\author{
Updated by ANNELI MIETTINEN
}

Population on 31 December

\begin{tabular}{llcccccccc}
\hline & \multicolumn{2}{l}{$\begin{array}{l}\text { Whole } \\
\text { country }\end{array}$} & \multicolumn{2}{c}{ Men } & \multicolumn{2}{c}{ Women } & \multicolumn{2}{c}{$\begin{array}{c}\text { Urban } \\
\text { municipalities }\end{array}$} & \multicolumn{2}{c}{$\begin{array}{c}\text { Rural } \\
\text { municipalities }\end{array}$} \\
& & 1000 & $\%$ & 1000 & $\%$ & 1000 & $\%$ & 1000 & $\%$ \\
\hline 1900 & 2655900 & 1311 & 49.3 & 1345 & 50.7 & 333 & 12.6 & 2323 & 87.4 \\
1910 & 2943400 & 1445 & 49.1 & 1499 & 50.9 & 432 & 14.7 & 2511 & 85.3 \\
1920 & 3147600 & 1533 & 48.7 & 1615 & 51.3 & 507 & 16.1 & 2640 & 83.9 \\
1930 & 3462700 & 1689 & 48.8 & 1774 & 51.2 & 715 & 20.6 & 2748 & 79.4 \\
1940 & 3695600 & 1793 & 48.5 & 1903 & 51.5 & 992 & 26.8 & 2704 & 73.2 \\
1950 & 4029800 & 1926 & 47.8 & 2104 & 52.2 & 1302 & 32.3 & 2727 & 67.7 \\
1960 & 4446200 & 2142 & 48.2 & 2304 & 51.8 & 1707 & 38.4 & 2739 & 61.6 \\
1970 & 4598300 & 2220 & 48.3 & 2378 & 51.7 & 2340 & 50.9 & 2258 & 49.1 \\
1980 & 4787800 & 2315 & 48.3 & 2473 & 51.7 & 2865 & 59.8 & 1923 & 40.2 \\
1990 & 4998500 & 2426 & 48.5 & 2572 & 51.5 & 3080 & 61.6 & 1919 & 38.4 \\
$2000^{*}$ & 5181100 & 2529 & 48.8 & 2652 & 51.2 & 3168 & 61.1 & 2013 & 38.9 \\
2002 & 5206300 & 2545 & 48.9 & 2661 & 51.1 & 3226 & 62.0 & 1980 & 38.0 \\
2003 & 5219700 & 2553 & 48.9 & 2667 & 51.1 & 3242 & 62.1 & 1977 & 37.9 \\
2004 & 5236600 & 2562 & 48.9 & 2675 & 51.1 & 3253 & 62.1 & 1983 & 37.9 \\
\hline
\end{tabular}

*The classification of municipalities into urban and rural areas changed in 1996. The division used here is the same as the one used by Statistics Finland.

Age structure (\%)

\begin{tabular}{rrrrrrrrrrr}
\hline & \multicolumn{3}{c}{ Whole population } & \multicolumn{3}{c}{ Men } & \multicolumn{3}{c}{ Women } \\
& $0-14$ & $15-64$ & $65-$ & $0-14$ & $15-64$ & $65-$ & $0-14$ & $15-64$ & $65-$ \\
\hline 1900 & 35.0 & 59.6 & 5.4 & 35.9 & 59.4 & 4.7 & 34.3 & 59.7 & 6.0 \\
1910 & 35.6 & 58.6 & 5.8 & 36.7 & 58.3 & 5.0 & 34.6 & 59.0 & 6.4 \\
1920 & 33.4 & 60.7 & 5.9 & 34.8 & 60.0 & 5.2 & 32.0 & 61.3 & 6.7 \\
1930 & 29.4 & 64.3 & 6.3 & 30.6 & 64.1 & 5.3 & 28.3 & 64.5 & 7.2 \\
1940 & 26.9 & 66.7 & 6.4 & 28.2 & 66.5 & 5.3 & 25.7 & 66.9 & 7.4 \\
1950 & 30.0 & 63.3 & 6.7 & 32.0 & 62.8 & 5.2 & 28.1 & 63.9 & 8.0 \\
1960 & 30.1 & 62.4 & 7.4 & 31.9 & 62.3 & 5.7 & 28.5 & 62.5 & 8.9 \\
1970 & 24.3 & 66.3 & 9.3 & 25.7 & 67.1 & 7.2 & 23.0 & 65.7 & 11.3 \\
1980 & 20.2 & 67.8 & 12.1 & 21.4 & 69.7 & 8.9 & 19.1 & 66.0 & 14.9 \\
1990 & 19.3 & 67.2 & 13.5 & 20.3 & 69.8 & 9.9 & 18.3 & 64.8 & 16.9 \\
2000 & 18.1 & 66.9 & 15.0 & 18.9 & 69.3 & 11.8 & 17.3 & 64.7 & 18.0 \\
2002 & 17.8 & 66.9 & 15.3 & 18.6 & 69.1 & 12.3 & 17.0 & 64.7 & 18.3 \\
2003 & 17.6 & 66.8 & 15.6 & 18.4 & 69.0 & 12.6 & 16.9 & 64.7 & 18.4 \\
2004 & 17.5 & 66.7 & 15.9 & 18.2 & 68.9 & 12.9 & 16.7 & 64.6 & 18.7 \\
\hline
\end{tabular}

${ }^{1}$ The data are based on the statistics of Statistics Finland, Helsinki. 
Age-specific fertility rate

\begin{tabular}{lllllllll}
\hline \multicolumn{7}{c}{} & \multicolumn{7}{c}{ Per 1,000 women } & & Total fertility \\
& $15-19$ & $20-24$ & $25-29$ & $30-34$ & $35-39$ & $40-44$ & $45-49$ & per woman \\
\hline $1901-10$ & 16.8 & 147.7 & 226.8 & 227.4 & 192.6 & 109.1 & 16.2 & 4.68 \\
$1911-20$ & 14.9 & 122.8 & 181.4 & 181.9 & 155.2 & 92.9 & 14.0 & 3.81 \\
$1921-30$ & 14.2 & 110.0 & 156.9 & 143.8 & 116.8 & 67.4 & 10.5 & 3.10 \\
$1931-40$ & 14.4 & 96.5 & 126.2 & 108.9 & 82.8 & 43.5 & 5.6 & 2.39 \\
$1941-45$ & 11.3 & 105.2 & 144.9 & 124.3 & 88.4 & 41.8 & 5.4 & 2.60 \\
$1946-50$ & 25.7 & 161.9 & 189.4 & 147.5 & 100.5 & 43.3 & 5.0 & 3.37 \\
$1951-55$ & 27.2 & 157.6 & 165.8 & 125.0 & 81.0 & 35.1 & 3.9 & 2.98 \\
$1956-60$ & 29.3 & 161.4 & 159.6 & 108.1 & 67.5 & 27.5 & 2.8 & 2.78 \\
$1961-65$ & 30.7 & 156.7 & 156.0 & 98.8 & 55.7 & 22.5 & 2.2 & 2.61 \\
$1966-70$ & 34.7 & 131.9 & 125.9 & 76.5 & 39.7 & 13.7 & 1.4 & 2.12 \\
$1971-75$ & 27.9 & 104.3 & 106.6 & 55.8 & 23.3 & 6.1 & 0.5 & 1.62 \\
$1976-80$ & 22.0 & 96.8 & 114.6 & 67.5 & 26.4 & 6.0 & 0.3 & 1.67 \\
$1981-85$ & 15.7 & 85.0 & 122.8 & 75.3 & 32.1 & 6.7 & 0.4 & 1.69 \\
$1986-90$ & 12.3 & 70.2 & 126.0 & 85.6 & 33.2 & 7.5 & 0.4 & 1.68 \\
$1991-95$ & 10.9 & 71.0 & 134.2 & 101.4 & 39.2 & 7.7 & 0.4 & 1.82 \\
$1996-00$ & 9.5 & 61.4 & 119.6 & 101.6 & 44.7 & 8.8 & 0.5 & 1.73 \\
2001 & 10.6 & 59.7 & 114.1 & 101.9 & 47.5 & 9.7 & 0.5 & 1.73 \\
2002 & 11.2 & 57.2 & 112.5 & 102.9 & 47.9 & 9.8 & 0.6 & 1.72 \\
2003 & 10.3 & 57.0 & 115.5 & 106.9 & 49.4 & 10.8 & 0.5 & 1.76 \\
2004 & 10.6 & 58.0 & 116.1 & 111.5 & 51.1 & 11.0 & 0.5 & 1.80 \\
\hline
\end{tabular}

Gross and net reproduction rate

\begin{tabular}{lccccc}
\hline & $\begin{array}{c}\text { Gross } \\
\text { reproduction } \\
\text { rate }\end{array}$ & $\begin{array}{c}\text { Net } \\
\text { reproduction } \\
\text { rate }\end{array}$ & . & $\begin{array}{c}\text { Gross } \\
\text { reproduction } \\
\text { rate }\end{array}$ & $\begin{array}{c}\text { Net } \\
\text { reproduction } \\
\text { rate }\end{array}$ \\
\hline $1901-10$ & 2.278 &. & $1971-75$ & 0.790 & 0.773 \\
$1911-20$ & 1.849 &.. & $1976-80$ & 0.814 & 0.801 \\
$1921-30$ & 1.504 &.. & $1981-85$ & 0.826 & 0.815 \\
$1931-35$ & 1.167 & 0.958 & $1986-90$ & 0.819 & 0.808 \\
$1936-40$ & 1.162 & 0.962 & $1991-95$ & 0.894 & 0.882 \\
$1941-45$ & 1.257 & 1.053 & $1996-00$ & 0.847 & 0.837 \\
$1946-50$ & 1.622 & 1.382 & 2001 & 0.844 & 0.837 \\
$1951-55$ & 1.452 & 1.373 & 2002 & 0.835 & 0.827 \\
$1956-60$ & 1.357 & 1.301 & 2003 & 0.864 & 0.856 \\
$1961-65$ & 1.276 & 1.236 & 2004 &.. &.. \\
$1966-70$ & 1.035 & 1.009 & & & \\
\hline
\end{tabular}


Median age of mother at first birth

\begin{tabular}{lccc}
\hline & Median age & & Median age \\
\hline $1961-65$ & 23.25 & $1991-95$ & 26.96 \\
$1966-70$ & 22.73 & $1996-00$ & 27.47 \\
$1971-75$ & 24.03 & 2001 & 27.24 \\
$1976-80$ & 25.18 & 2002 & 27.37 \\
$1981-85$ & 25.40 & 2003 & 27.59 \\
$1986-90$ & 26.30 & 2004 &.. \\
\hline
\end{tabular}

Live births

\begin{tabular}{cccccc}
\hline & $\begin{array}{c}\text { Annual mean/ } \\
\text { number }\end{array}$ & $\begin{array}{c}\text { Per 1,000 } \\
\text { of mean } \\
\text { population }\end{array}$ & & $\begin{array}{c}\text { Annual mean/ } \\
\text { number }\end{array}$ & $\begin{array}{c}\text { Per 1,000 } \\
\text { of mean } \\
\text { population }\end{array}$ \\
\hline $1901-10$ & 90292 & 32.4 & $1981-85$ & 64868 & 13.4 \\
$1911-20$ & 83045 & 27.0 & $1986-90$ & 62534 & 12.6 \\
$1921-30$ & 78184 & 23.6 & $1991-95$ & 65050 & 12.8 \\
$1931-40$ & 70584 & 19.7 & $1996-00$ & 58295 & 11.3 \\
$1941-50$ & 92614 & 24.2 & 2001 & 56189 & 10.8 \\
$1951-60$ & 88024 & 20.8 & 2002 & 55555 & 10.7 \\
$1961-70$ & 76466 & 16.8 & 2003 & 56630 & 10.9 \\
$1971-80$ & 62789 & 13.3 & 2004 & 57758 & 11.0 \\
\hline
\end{tabular}

Illegitimate live births

\begin{tabular}{cccccc}
\hline & $\begin{array}{c}\text { Annual mean/ } \\
\text { number }\end{array}$ & $\begin{array}{c}\text { Percent of } \\
\text { live births }\end{array}$ & & $\begin{array}{c}\text { Annual mean/ } \\
\text { number }\end{array}$ & $\begin{array}{c}\text { Percent of } \\
\text { live births }\end{array}$ \\
\hline $1901-10$ & 6120 & 6.8 & $1981-85$ & 9388 & 14.5 \\
$1911-20$ & 6573 & 6.8 & $1986-90$ & 13301 & 21.3 \\
$1921-30$ & 6606 & 8.4 & $1991-95$ & 19628 & 30.2 \\
$1931-40$ & 5349 & 7.6 & $1996-00$ & 21781 & 37.4 \\
$1941-50$ & 5629 & 6.1 & 2001 & 22222 & 39.5 \\
$1951-60$ & 3819 & 4.3 & 2002 & 22156 & 39.9 \\
$1961-70$ & 3621 & 4.7 & 2003 & 22649 & 40.0 \\
$1971-80$ & 6182 & 9.8 & 2004 & 23554 & 40.8 \\
\hline
\end{tabular}

Abortions

\begin{tabular}{lcclcc}
\hline & $\begin{array}{c}\text { Annual mean/ } \\
\text { number }\end{array}$ & $\begin{array}{c}\text { Percent of } \\
\text { live births }\end{array}$ & & $\begin{array}{c}\text { Annual mean/ } \\
\text { number }\end{array}$ & $\begin{array}{c}\text { Percent of } \\
\text { live births }\end{array}$ \\
\hline 1970 & 14757 & 22.9 & $1996-00$ & 10637 & 18.3 \\
$1971-75$ & 22105 & 36.3 & 2001 & 10699 & 19.0 \\
$1976-80$ & 17081 & 26.4 & 2002 & 10902 & 19.6 \\
$1981-85$ & 13764 & 21.2 & 2003 & 10643 & 18.8 \\
$1986-90$ & 12791 & 20.5 & $2004^{*}$ & 10724 & 18.6 \\
$1991-95$ & 10610 & 16.3 & & & \\
\hline
\end{tabular}

* Preliminary data 
Marriages

\begin{tabular}{|c|c|c|c|c|c|c|c|}
\hline & \multicolumn{2}{|c|}{$\begin{array}{l}\text { Annual mean/ } \\
\text { number }\end{array}$} & \multirow[t]{2}{*}{$\begin{array}{l}\text { Per } 1,000 \\
\text { of mean } \\
\text { population }\end{array}$} & & \multicolumn{2}{|c|}{$\begin{array}{l}\text { Annual mean/ } \\
\text { number }\end{array}$} & \multirow[t]{2}{*}{$\begin{array}{l}\text { Per } 1,000 \\
\text { of mean } \\
\text { population }\end{array}$} \\
\hline & Marriages & $\begin{array}{c}\text { Registered } \\
\text { unions }\end{array}$ & & & Marriages & $\begin{array}{c}\text { Registered } \\
\text { unions }\end{array}$ & \\
\hline $\begin{array}{l}1901-10 \\
1911-20\end{array}$ & 18947 &.. & 6.8 & $\begin{array}{l}1986-90 \\
100105\end{array}$ & 25516 &.. & 5.2 \\
\hline 1921-30 & 23725 &... & 7.2 & 1996-00 & 24470 &.. & $\begin{array}{l}4.0 \\
4.7\end{array}$ \\
\hline $1931-40$ & 28491 & .. & 7.9 & 2001 & 24830 & .. & 4.8 \\
\hline $1941-50$ & 37367 & .. & 9.8 & $2002^{*}$ & 26969 & 446 & 5.2 \\
\hline $1951-60$ & 32191 &.. & 7.6 & 2003 & 25815 & 190 & 5.0 \\
\hline $1961-70$ & 37398 & .. & 8.2 & 2004 & 29342 & 186 & 5.6 \\
\hline $1971-80$ & 32575 &.. & 7.0 & & & & \\
\hline $1981-85$ & 28867 & .. & 5.9 & & & & \\
\hline
\end{tabular}

* Since 2002, same-sex couples have been able to register their union.

Median age at first marriage

\begin{tabular}{lccccc}
\hline & Men & Women & & Men & Women \\
\hline $1901-05$ & 26.1 & 23.5 & $1961-65$ & 24.1 & 22.3 \\
$1906-10$ & 26.2 & 23.6 & $1966-70$ & 23.6 & 22.2 \\
$1911-15$ & 26.4 & 23.7 & $1971-75$ & 24.0 & 22.7 \\
$1916-20$ & 27.0 & 24.0 & $1976-80$ & 25.2 & 23.3 \\
$1921-25$ & 26.9 & 24.0 & $1981-85$ & 26.2 & 24.2 \\
$1926-30$ & 26.6 & 23.9 & $1986-90$ & 27.1 & 25.2 \\
$1931-35$ & 27.1 & 24.4 & $1991-95$ & 28.1 & 26.3 \\
$1936-40$ & 27.6 & 24.7 & $1996-00$ & 29.3 & 27.1 \\
$1941-45$ & 27.6 & 24.3 & 2001 & 29.6 & 27.3 \\
$1946-50$ & 26.1 & 23.7 & 2002 & 29.8 & 27.7 \\
$1951-55$ & 24.9 & 23.2 & 2003 & 29.9 & 27.8 \\
$1956-60$ & 24.6 & 22.8 & 2004 & 30.4 & 28.2 \\
\hline
\end{tabular}

\section{Consensual unions}

Persons living in consensual union of all persons living in union $(\%)^{*}$

\begin{tabular}{lccccccc} 
& $15-24$ & $25-29$ & $30-34$ & $35-44$ & $45-64$ & $15-64$ & Number \\
\hline 1981 & 44.6 & 19.4 & 12.4 & 6.5 & 2.5 & 10.3 & 214000 \\
1985 & 55.0 & 27.1 & 13.5 & 7.4 & 4.4 & 12.8 & 283000 \\
1990 & 69.7 & 39.3 & 21.6 & 12.4 & 6.8 & 21.3 & 366580 \\
1995 & 77.4 & 47.2 & 28.9 & 17.3 & 8.8 & 21.0 & 430444 \\
2000 & 81.8 & 54.6 & 34.8 & 22.7 & 11.4 & 24.8 & 509456 \\
2002 & 82.9 & 56.8 & 36.3 & 24.4 & 12.5 & 26.1 & 537638 \\
2003 & 83.5 & 57.0 & 36.9 & 25.1 & 13.1 & 26.7 & 548270 \\
\hline
\end{tabular}

*Until 1990, estimation by Statistics Finland on the basis of information from Labor Force Surveys. Since 1990, consensual unions are derived from population registers

Divorces

\begin{tabular}{lccccc}
\hline & $\begin{array}{c}\text { Annual mean/ } \\
\text { number }\end{array}$ & $\begin{array}{c}\text { Per 1,000 } \\
\text { of mean } \\
\text { population }\end{array}$ & $\begin{array}{c}\text { Annual mean/ } \\
\text { number }\end{array}$ & $\begin{array}{c}\text { Per 1,000 } \\
\text { of mean } \\
\text { population }\end{array}$ & $\begin{array}{c}\text { Per 1,000 } \\
\text { of mean } \\
\text { population }\end{array}$ \\
\hline $1931-40$ & 1391 & 0.4 & $1991-95$ & 13260 & 2.6 \\
$1941-50$ & 3721 & 1.0 & $1996-00$ & 13819 & 2.7 \\
$1951-60$ & 3584 & 0.8 & 2001 & 13589 & 2.6 \\
$1961-70$ & 4860 & 1.1 & $2002^{*}$ & 13360 & 2.6 \\
$1971-80$ & 9389 & 2.0 & 2003 & 13475 & 2.6 \\
$1981-85$ & 9538 & 2.0 & 2004 & 13234 & 2.5 \\
$1986-90$ & 11898 & 2.4 & & & \\
\hline
\end{tabular}

"Since 2002, divorce from a registered same-sex couple is included in the data 
Families with children under 18 years of age by type of family (\%)

\begin{tabular}{ccccccc}
\hline & $\begin{array}{c}\text { Married } \\
\text { couples with } \\
\text { children }^{1}\end{array}$ & $\begin{array}{c}\text { Couples in con- } \\
\text { sensual union } \\
\text { with children }\end{array}$ & $\begin{array}{c}\text { Mothers with } \\
\text { children }\end{array}$ & $\begin{array}{c}\text { Fathers with } \\
\text { children }\end{array}$ & $\begin{array}{c}\text { Registered } \\
\text { couples with } \\
\text { children }\end{array}$ & Number \\
\hline 1950 & 85.9 &.. & 12.4 & 1.7 &.. & 599329 \\
1960 & 88.7 &.. & 9.9 & 1.3 &. & 678046 \\
1970 & 88.9 & 0.9 & 9.0 & 1.2 &.. & 677035 \\
1980 & 83.1 & 4.7 & 10.9 & 1.4 &.. & 688732 \\
1990 & 76.6 & 9.4 & 12.3 & 1.7 &.. & 640637 \\
1995 & 70.2 & 12.3 & 15.4 & 2.1 &.. & 639610 \\
2000 & 65.1 & 15.5 & 17.0 & 2.4 &.. & 612627 \\
2002 & 63.7 & 16.6 & 17.2 & 2.4 & 0.0 & 598917 \\
2003 & 63.0 & 17.1 & 17.4 & 2.5 & 0.0 & 595027 \\
2004 & 62.6 & 17.4 & 17.4 & 2.5 & 0.0 & 592809 \\
\hline
\end{tabular}

${ }^{1}$ Includes not-married couples with children under 18 years, until 1970. Consensual unions are estimated on the basis of population survey (1970) and on population registers (since 1980).

Families with children under 18 years of age by type of family and number of children (\%)

\begin{tabular}{|c|c|c|c|c|c|}
\hline & \multicolumn{3}{|c|}{ Number of children } & \multirow[b]{2}{*}{$4+$} & \multirow[b]{2}{*}{ Number } \\
\hline & 1 & 2 & 3 & & \\
\hline \multicolumn{6}{|c|}{ Married cuoples with children ${ }^{1}$} \\
\hline 1960 & 34.4 & 30.7 & 17.5 & 17.4 & 601542 \\
\hline 1970 & 40.2 & 33.9 & 15.5 & 10.4 & 602076 \\
\hline 1980 & 44.6 & 41.1 & 11.1 & 3.2 & 588373 \\
\hline 1990 & 38.9 & 42.4 & 14.5 & 4.1 & 490965 \\
\hline 2000 & 37.3 & 40.4 & 16.5 & 5.8 & 398892 \\
\hline 2002 & 36.8 & 40.6 & 16.7 & 6.0 & 381340 \\
\hline 2003 & 36.5 & 40.8 & 16.7 & 6.0 & 374940 \\
\hline 2004 & 36.2 & 40.9 & 16.7 & 6.1 & 371323 \\
\hline \multicolumn{6}{|c|}{ Couples living in consensual union with children } \\
\hline 1990 & 60.0 & 30.2 & 7.9 & 1.9 & 59827 \\
\hline 2000 & 51.5 & 35.8 & 10.0 & 2.6 & 95120 \\
\hline 2002 & 50.7 & 36.4 & 10.2 & 2.7 & 99625 \\
\hline 2003 & 50.5 & 36.7 & & .. & 101742 \\
\hline 2004 & 50.8 & 36.4 & 10.2 & 2.6 & 103214 \\
\hline \multicolumn{6}{|c|}{ Mothers with children } \\
\hline 1960 & 61.1 & 23.0 & 9.2 & 6.7 & 67381 \\
\hline 1970 & 60.7 & 23.8 & 9.5 & 6.0 & 66303 \\
\hline 1980 & 67.7 & 25.3 & 5.6 & 1.4 & 88896 \\
\hline 1990 & 65.6 & 27.2 & 5.9 & 1.3 & 78869 \\
\hline 2000 & 58.0 & 30.9 & 8.6 & 2.5 & 103984 \\
\hline 2002 & 57.2 & 31.2 & 8.9 & 2.7 & 103286 \\
\hline 2003 & 57.1 & 31.3 & 8.9 & 2.7 & 103446 \\
\hline 2004 & 57.0 & 31.4 & 8.9 & 2.7 & 103376 \\
\hline \multicolumn{6}{|c|}{ Fathers with children } \\
\hline 1960 & 56.7 & 24.8 & 10.3 & 8.2 & 9123 \\
\hline 1970 & 61.1 & 24.3 & 9.9 & 5.6 & 8656 \\
\hline 1980 & 71.1 & 23.0 & 4.8 & 1.1 & 11463 \\
\hline 1990 & 71.7 & 23.4 & 4.1 & 0.8 & 11074 \\
\hline 2000 & 69.1 & 24.6 & 5.2 & 1.1 & 14631 \\
\hline 2002 & 68.1 & 25.2 & 5.4 & 1.3 & 14634 \\
\hline 2003 & 68.1 & 25.3 & 5.4 & 1.2 & 14852 \\
\hline 2004 & 67.3 & 25.9 & 5.5 & 1.2 & 14837 \\
\hline \multicolumn{6}{|c|}{ Registered couples with children } \\
\hline 2002 & 59.4 & 28.1 & 12.5 & 0.0 & 32 \\
\hline 2003 & 59.6 & 27.7 & 12.8 & 0.0 & 47 \\
\hline 2004 & 55.9 & 35.6 & 8.5 & 0.0 & 59 \\
\hline
\end{tabular}

${ }^{1}$ Includes not-married couples with children under 18 years, until 1970. Consensual unions are estimated on the basis of population survey (1970) and on population registers (since 1980). 
Deaths

\begin{tabular}{cccccccc}
\hline & $\begin{array}{c}\text { Annual } \\
\text { mean/ } \\
\text { number }\end{array}$ & $\begin{array}{c}\text { Per 1,000 } \\
\text { of mean } \\
\text { population }\end{array}$ & $\begin{array}{c}\text { Infant } \\
\text { mortality* }\end{array}$ & & $\begin{array}{c}\text { Annual } \\
\text { mean/ } \\
\text { number }\end{array}$ & $\begin{array}{c}\text { Per 1,000 } \\
\text { of mean } \\
\text { population }\end{array}$ & $\begin{array}{c}\text { Infant } \\
\text { mortality }\end{array}$ \\
\hline $1901-10$ & 52099 & 18.7 & 123.8 & $1986-90$ & 48663 & 9.8 & 5.9 \\
$1911-20$ & 58284 & 19.0 & 111.8 & $1991-95$ & 49481 & 9.8 & 4.8 \\
$1921-30$ & 49573 & 14.9 & 91.8 & $1996-00$ & 49244 & 9.6 & 3.9 \\
$1931-40$ & 50230 & 14.0 & 72.1 & 2001 & 48550 & 9.4 & 3.2 \\
$1941-50$ & 51838 & 13.6 & 56.6 & 2002 & 49418 & 9.5 & 3.0 \\
$1951-60$ & 39381 & 9.3 & 28.4 & 2003 & 48996 & 9.4 & 3.1 \\
$1961-70$ & 43494 & 9.5 & 16.6 & 2004 & 47600 & 9.1 & 3.3 \\
$1971-80$ & 44243 & 9.4 & 9.7 & & & & \\
$1981-85$ & 45299 & 9.3 & 6.3 & & & & \\
\hline
\end{tabular}

${ }^{*}$ Mortality during the first year of life per 1000 live births

\section{Life expectancy}

\begin{tabular}{lcclcc}
\hline & Men & Women & & Men & Women \\
\hline $1911-20^{1}$ & 43.4 & 49.1 & $1976-80$ & 68.5 & 77.2 \\
$1921-30$ & 50.7 & 55.1 & $1981-85$ & 70.1 & 78.4 \\
$1931-40^{2}$ & 54.5 & 59.6 & $1986-90$ & 70.7 & 78.8 \\
$1941-45^{2}$ & 54.6 & 61.1 & $1991-95$ & 72.1 & 79.7 \\
$1946-50$ & 58.6 & 65.8 & $1996-00$ & 73.5 & 80.8 \\
$1951-55$ & 63.4 & 69.8 & 2001 & 74.6 & 81.5 \\
$1956-60$ & 65.9 & 71.6 & 2002 & 74.9 & 81.5 \\
$1961-65$ & 65.4 & 72.6 & 2003 & 75.1 & 81.8 \\
$1966-70$ & 65.9 & 73.6 & 2004 & 75.3 & 82.3 \\
$1971-75$ & 66.7 & 75.2 & & & \\
\hline
\end{tabular}

${ }^{1}$ Including deaths due to war operations

${ }^{2}$ Excluding deaths due to war operations

\section{Excess of births over deaths}

\begin{tabular}{lccccc}
\hline & $\begin{array}{c}\text { Annual } \\
\text { mean/ } \\
\text { number }\end{array}$ & $\begin{array}{c}\text { Per 1,000 } \\
\text { of mean } \\
\text { population }\end{array}$ & & $\begin{array}{c}\text { Annual } \\
\text { mean/ } \\
\text { number }\end{array}$ & $\begin{array}{c}\text { Per } 1,000 \\
\text { of mean } \\
\text { population }\end{array}$ \\
\hline $1901-10$ & 38194 & 13.7 & $1981-85$ & 19569 & 4.0 \\
$1911-20$ & 24760 & 8.1 & $1986-90$ & 13871 & 2.8 \\
$1921-30$ & 28611 & 8.7 & $1991-95$ & 15569 & 3.1 \\
$1931-40$ & 20355 & 5.7 & $1996-00$ & 9051 & 1.8 \\
$1941-50$ & 40776 & 10.6 & 2001 & 7639 & 1.5 \\
$1951-60$ & 48643 & 11.5 & 2002 & 6137 & 1.2 \\
$1961-70$ & 32972 & 7.2 & 2003 & 7634 & 1.5 \\
$1971-80$ & 18546 & 3.9 & 2004 & 10158 & 1.9 \\
\hline
\end{tabular}


International migration

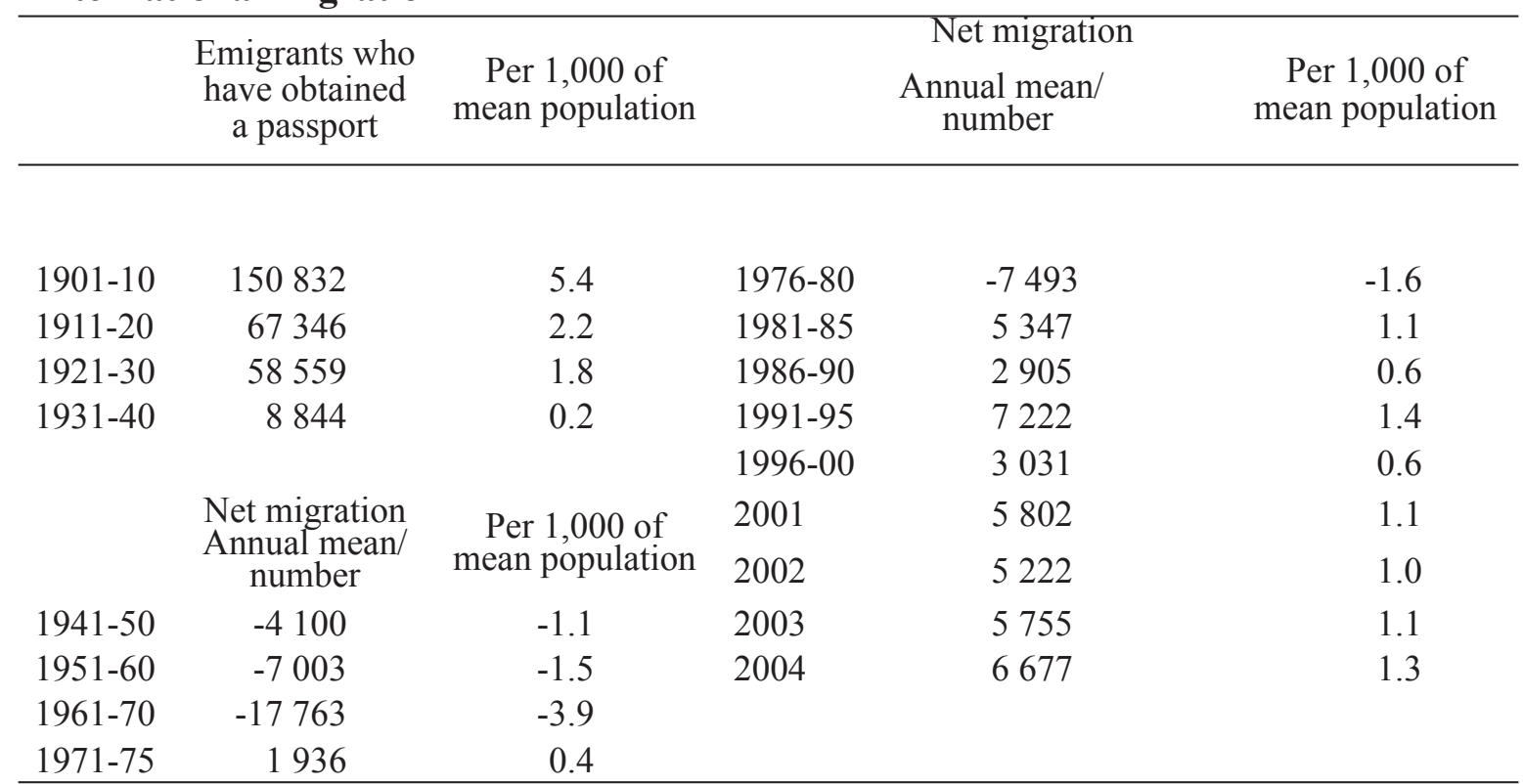

Internal migration

\begin{tabular}{cccccc}
\hline & $\begin{array}{c}\text { Annual mean/ } \\
\text { number }^{*}\end{array}$ & $\begin{array}{c}\text { Percent of mean } \\
\text { population }\end{array}$ & $\begin{array}{c}\text { Annual mean/ } \\
\text { number }\end{array}$ & $\begin{array}{c}\text { Percent of mean } \\
\text { population }\end{array}$ \\
\hline $1901-10$ & 74784 & 2.7 & $1981-85$ & 193681 & 4.0 \\
$1911-20$ & 85111 & 2.8 & $1986-90$ & 200737 & 4.1 \\
$1921-30$ & 79248 & 2.4 & $1991-95$ & 189999 & 3.8 \\
$1931-39$ & 136470 & 3.8 & $1996-00$ & 247151 & 4.8 \\
$1941-50$ & 132778 & 3.5 & 2001 & 280140 & 5.4 \\
$1951-60$ & 172098 & 4.0 & 2002 & 272023 & 5.2 \\
$1961-70$ & 219330 & 4.8 & 2003 & 271333 & 5.2 \\
$1971-80$ & 213569 & 4.6 & 2004 & 281976 & 5.4 \\
\hline
\end{tabular}

*Average in-migration to rural and urban municipalities

\section{Foreigners}

\begin{tabular}{rcclr}
\hline & $\begin{array}{c}\text { Number of } \\
\text { foreigners }\end{array}$ & $\begin{array}{c}\text { Percent of } \\
\text { total population }\end{array}$ & Groups of foreigners by citizenship in 2003 \\
\hline 1976 & 12154 & 0.3 & Europe & 72635 \\
1980 & 12502 & 0.3 & EU-countries & 18341 \\
1985 & 16478 & 0.3 & of which Sweden & 8124 \\
1990 & 26255 & 0.5 & Russia & 24998 \\
1995 & 68566 & 1.3 & Estonia & 13397 \\
2000 & 91074 & 1.4 & Other Europe & 15899 \\
2001 & 98577 & 1.6 & Africa & 9045 \\
2002 & 103682 & 1.6 & of which Somalia & 4642 \\
2003 & 107003 & 1.6 & North America & 2807 \\
2004 & 108346 & 2.1 & South America & 1397 \\
& & & Asia and Oceania & 19742 \\
& & & Unknown/without citizenship & 1377 \\
\hline
\end{tabular}


Economically active population aged $15-64$ by industry (\%)

\begin{tabular}{lrrrrrrr}
\hline & 1950 & 1960 & 1970 & 1980 & $1990^{1}$ & 2000 & 2003 \\
\hline Agriculture and forestry, fishing & 45.8 & 35.5 & 20.3 & 12.6 & 8.5 & 6.1 & 5.1 \\
and hunting & 20.8 & 21.6 & 25.9 & 26.3 & 21.9 & 21.2 & 19.9 \\
Manufacturing, etc. & 6.3 & 8.7 & 8.3 & 7.1 & 7.0 & 6.4 & 6.4 \\
Construction & 9.5 & 13.6 & 18.9 & 19.1 & 26.3 & 27.5 & 28.5 \\
Trade, etc. & 5.4 & 6.3 & 7.1 & 7.9 & 7.2 & 7.4 & 7.3 \\
Transport and communications & 10.8 & 14.0 & 18.1 & 24.8 & 26.8 & 31.3 & 32.4 \\
Services & 1.4 & 0.3 & 1.4 & 2.2 & 2.3 & 0.3 & 0.3 \\
Unknown & 1960 & 2033 & 2118 & 2222 & 2332 & 2335 & 2365 \\
1000 persons & & & & & & 23 & \\
\hline
\end{tabular}

${ }^{1}$ Employed labor force. The grounds for classification have been changed in some extent in 1989 and in 1995.

Females in labor force

The proportion of females
in labor force $(\%)$ Female labor force as a percentage of female population aged

\begin{tabular}{lcccc} 
& & $20-44 \mathrm{yrs}$ & $15-64 \mathrm{yrs}$ & $15-74 \mathrm{yrs}$ \\
\hline $1960-64$ & 44.0 &.. &.. & 56.2 \\
$1965-69$ & 43.3 &.. &.. & 55.1 \\
$1970-74$ & 44.7 &.. &.. & 57.9 \\
$1975-79$ & 46.3 & 78.0 & 66.8 & 59.9 \\
$1980-84$ & 47.3 & 82.5 & 71.2 & 63.0 \\
$1985-89$ & 47.7 & 84.2 & 72.8 & 64.4 \\
$1990-94$ & 47.5 & 80.6 & 70.8 & 62.2 \\
$1995-99$ & 47.5 & 79.0 & 70.4 & 61.6 \\
2000 & 47.9 & 79.9 & 72.0 & 63.2 \\
2001 & 47.9 & 80.0 & 72.4 & 63.6 \\
2002 & 48.2 & 80.8 & 72.8 & 64.0 \\
2003 & 48.0 & 80.0 & 72.1 & 63.5 \\
2004 & 48.1 & 78.9 & 72.0 & 63.3 \\
\hline
\end{tabular}

Unemployment rate

\begin{tabular}{lccccc}
\hline & Both sexes & Men & $\begin{array}{c}\text { Men aged } \\
15-24\end{array}$ & Women & $\begin{array}{c}\text { Women aged } \\
15-24\end{array}$ \\
\hline $1965-69$ & 2.5 & 3.4 &.. & 1.3 &.. \\
$1970-74$ & 2.3 & 2.6 & 4.7 & 1.8 & 3.8 \\
$1975-79$ & 5.1 & 5.8 & 11.8 & 4.3 & 9.1 \\
$1980-84$ & 5.1 & 5.3 & 10.4 & 4.9 & 9.9 \\
$1985-89$ & 4.7 & 5.2 & 9.7 & 4.2 & 7.9 \\
$1990-94$ & 12.1 & 13.7 & 25.8 & 10.3 & 19.3 \\
$1995-99^{1}$ & 13.9 & 13.7 & 25.8 & 14.2 & 25.2 \\
2000 & 9.8 & 9.1 & 21.1 & 10.6 & 21.6 \\
2001 & 9.1 & 8.6 & 19.6 & 9.7 & 20.0 \\
2002 & 9.1 & 9.1 & 21.2 & 9.1 & 20.9 \\
2003 & 9.0 & 9.2 & 21.9 & 8.9 & 21.6 \\
2004 & 8.8 & 8.7 & 22.0 & 8.9 & 19.4 \\
\hline
\end{tabular}

${ }^{1}$ The grounds for classification of unemployment have been changed somewhat in 1997. 